ISSN 1420-3049

www.mdpi.com/journal/molecules

Review

\title{
Review of Platensimycin and Platencin: Inhibitors of $\beta$-Ketoacyl-acyl Carrier Protein (ACP) Synthase III (FabH)
}

\author{
Ruofeng Shang ${ }^{1}$, Jianping Liang ${ }^{1}$, Yunpeng Yi ${ }^{1}$, Yu Liu ${ }^{1}$ and Jiatu Wang ${ }^{2, *}$
}

1 Key Laboratory of New Animal Drug Project of Gansu Province, Key Laboratory of Veterinary Pharmaceutical Development, Ministry of Agriculture, Lanzhou Institute of Husbandry and Pharmaceutical Sciences of CAAS, Lanzhou 730050, China; E-Mails: shangrf1974@163.com (R.S.); liangjianping@caas.cn (J.L.); yiyunpeng88@163.com (Y.Y.); yangguang8684@163.com (Y.L.)

2 Affiliated Hospital of Gansu University of Chinese Medicine, Lanzhou 730000, China

* Authors to whom correspondence should be addressed; E-Mails: Wang_jiatu@sina.com; Tel.: +86-931-863-5283; Fax: +86-931-863-5229.

Academic Editor: Peter J. Rutledge

Received: 9 July 2015 / Accepted: 28 August 2015 / Published: 3 September 2015

\begin{abstract}
Platensimycin and platencin were successively discovered from the strain Streptomyces platensis through systematic screening. These natural products have been defined as promising agents for fighting multidrug resistance in bacteria by targeting type II fatty acid synthesis with slightly different mechanisms. Bioactivity studies have shown that platensimycin and platencin offer great potential to inhibit many resistant bacteria with no cross-resistance or toxicity observed in vivo. This review summarizes the general information on platensimycin and platencin, including antibacterial and self-resistant mechanisms. Furthermore, the total synthesis pathways of platensimycin and platencin and their analogues from recent studies are presented.
\end{abstract}

Keywords: platensimycin; platencin; drug resistance; antibacterial activities; synthesis; analogues

\section{Introduction}

Many available drugs have reduced or lost their curative effect, leading to increased morbidity and mortality, because of the emergence and spread of multidrug resistant bacteria [1]. The drastic increase 
in pathogenic bacterial resistance, especially in multiresistant bacteria, is one of the most serious problems endangering human health and urgently needs for effective solutions [2,3]. Chemical modifications of existing scaffolds have afforded antibiotics with improved activity and have served well the development of new and effective antibiotics in past decades. However, such modifications are becoming increasingly challenging [4]. Therefore, the discovery of novel antibiotic chemical scaffolds with new modes of action to overcome drug resistance is crucial [5]. Natural products are an important tool for this approach, particularly platensimycin (1) and platencin (2) (Figure 1).

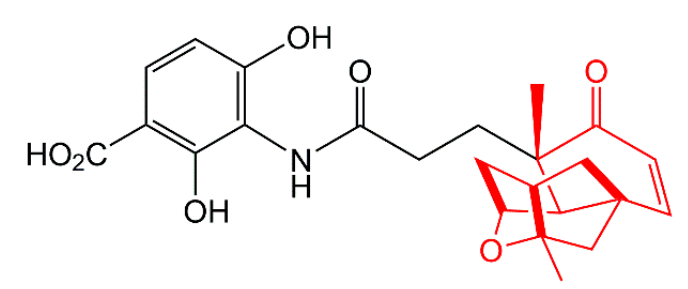

1

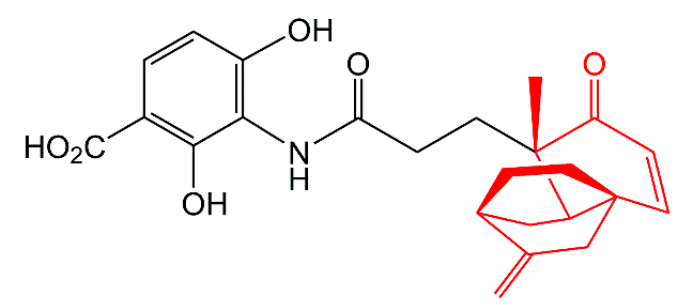

2

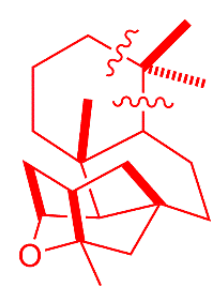

ent-Kaurene

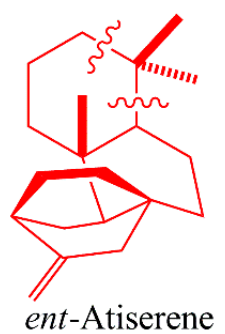

Figure 1. Structures of platensimycin 1 and platencin 2 and their biosynthetic relationship to ent-kaurene and ent-atiserene.

The natural products platensimycin and platencin, discovered by employing a novel antisense differential sensitivity screening strategy, were reported recently from soil bacterial strains of Streptomyces platensis [6-8]. Platensimycin inhibits fatty acid acyl carrier protein synthase II (FabF) selectively [6], whereas platencin is a balanced dual inhibitor of both FabF and fatty acid acyl carrier protein synthase III (FabH) [9]. Because of their unique antibacterial mechanism, these natural products show potent, broad-spectrum Gram-positive in vitro activity, including against antibiotic-resistant bacteria, such as methicillin-resistant Staphylococcus aureus (MRSA), vancomycin-intermediate S. aureus (VISA), and vancomycin-resistant Enterococci (VRE) [4]. Importantly, both platensimycin and platencin exhibit no cross-resistance to clinically relevant pathogens and show in vivo efficacy without toxicity [10].

This review will provide an overview of the isolation, antibacterial activities, biosynthetic machineries, and antibacterial and self-resistant mechanisms of platensimycin and platencin. Moreover, total synthesis and their analogues in recent years are also described.

\section{Isolation, Antibacterial Activities, and Biosynthesis}

Platensimycin was first discovered by the Merck research group through a systematic screening of approximately 250,000 natural product extracts as part of their target-based whole-cell screening strategy using an antisense differential sensitivity assay [11]. This product was isolated $(2-4 \mathrm{mg} / \mathrm{L})$ from 
the fermentation broth of Streptomyces platensis (MA7327 and MA7331) by a two-step process, a capture step followed by reversed-phase high performance liquid chromatography (HPLC), and the structure was elucidated by 2D nuclear magnetic resonance (NMR) methods and confirmed by X-ray crystallographic analysis [11]. Platensimycin was ultimately proven to be a new class of antibiotics with no cross-resistance in other classes of antibiotic-resistant bacteria [12,13]. It is a selective inhibitor for $S$. aureus FabF and Escherichia coli (E. coli) FabF/B enzymes with IC $_{50}$ values of 48 and $160 \mathrm{~nm}$, respectively [14]. In vitro antibacterial studies have shown that platensimycin is effective against Gram-positive bacteria with minimal inhibitory concentration (MIC) values of $0.1-0.32 \mathrm{mg} / \mathrm{mL}$ [15].

Another extract of a new strain of Streptomyces platensis (MA7339) was isolated from a soil sample and identified by the same research group after the discovery of platensimycin. Platencin was discovered and isolated by bioassay-guided fractionation of this extract $[4,16]$. Unlike platensimycin, platencin possesses a polycyclic enone skeleton bearing an exocyclic double bond instead of an ether linkage, and is a balanced dual inhibitor of both FabH $\left(\mathrm{IC}_{50}=9.2 \mathrm{~mm}\right)$ and FabF $\left(\mathrm{IC}_{50}=4.6 \mathrm{~mm}\right)$. Platencin shows broad-spectrum antibacterial activity against various bacterial strains, including drug resistant bacteria such as MRSA, linezolid-resistant $S$. aureus, vancomycin intermediate $S$. aureus and VRE with MIC values $\geq 0.06-4 \mathrm{mg} / \mathrm{mL}[14]$.

Feeding experiments using ${ }^{13} \mathrm{C}$ precursors suggest that the aminobenzoic acid moieties of platensimycin and platencin were biosynthesized from pyruvate and acetate via the tricarboxylic acid cycle and phosphoenolpyruvate in the strains of Streptomyces platensis. The C-17 polycyclic enone acid moiety of platensimycin was biosynthesized via a non-mevalonate terpenoid pathway by the oxidative excision of three carbons from ent-kaurene (Figure 1) [17,18]. Recent studies showed that the biosynthesis of platencin in Streptomyces platensis MA7327 and MA7339 is controlled by ent-atiserene (Figure 1) synthases, a new pathway for diterpenoid biosynthesis that is different from ent-kaurene synthases [19].

\section{Antibacterial and Self-Resistant Mechanism}

Fatty acids form the building blocks of many cellular structures and are required for energy storage in bacteria [9]. The synthesis of fatty acids in bacteria is conducted by type II bacterial fatty acid synthesis (FASII) which is different from the synthesis found in eukaryotes (mammals and fungi; type I). Type II FAS consists of many discrete enzymes- involved in condensation, reduction and dehydration [20]. Platensimycin and platencin interfere with the synthesis of fatty acids by selectively inhibiting FabF, an elongation-condensing enzyme whose main function is to add acetate units to the growing fatty acid chain [9]. A FabH/FabF PAGE elongation assay with the crude $S$. aureus cytosolic proteins, which closely mimics the events inside living cells, suggested that platensimycin is a selective FabF inhibitor, whereas platencin is a dual inhibitor with similar inhibition efficiency for both FabF and FabH (Figure 2) and thus inhibits fatty acid synthesis in S. aureus through a synergistic effect [4].

Pathogens have generally gained their resistance genes by horizontal gene transfer from non-pathogenic bacteria, with one potential source being antibiotic producing bacteria that developed highly effective mechanisms to avoid suicide [21]. Therefore, it is important to understand the self-resistance mechanisms within platensimycin and platencin producing organisms. A standard disk diffusion assay identified the $p t m P 3$ or $p t n P 3$ gene within the platensimycin or platencin biosynthetic 
cluster in the S. platensis MA7327 and MA7339 strains as the major resistance conferring element. The FabF gene within the housekeeping fatty acid synthase locus was identified as the second resistance conferring element. PtmP3/PtnP3 and FabF, therefore, confer platensimycin and platencin resistance by target replacement (i.e., FabF and FabH by $\mathrm{PtmP} 3$ ) and target modification (i.e., a platensimycin-insensitive variant of FabF), respectively (Figure 2). PtmP3/PtnP3 also represents an unprecedented mechanism for fatty acid biosynthesis in which $\mathrm{FabH}$ and $\mathrm{FabF}$ are functionally replaced by a single condensing enzyme (i.e., a platensimycin-insensitive variant of $\mathrm{FabF}$ ) [10].

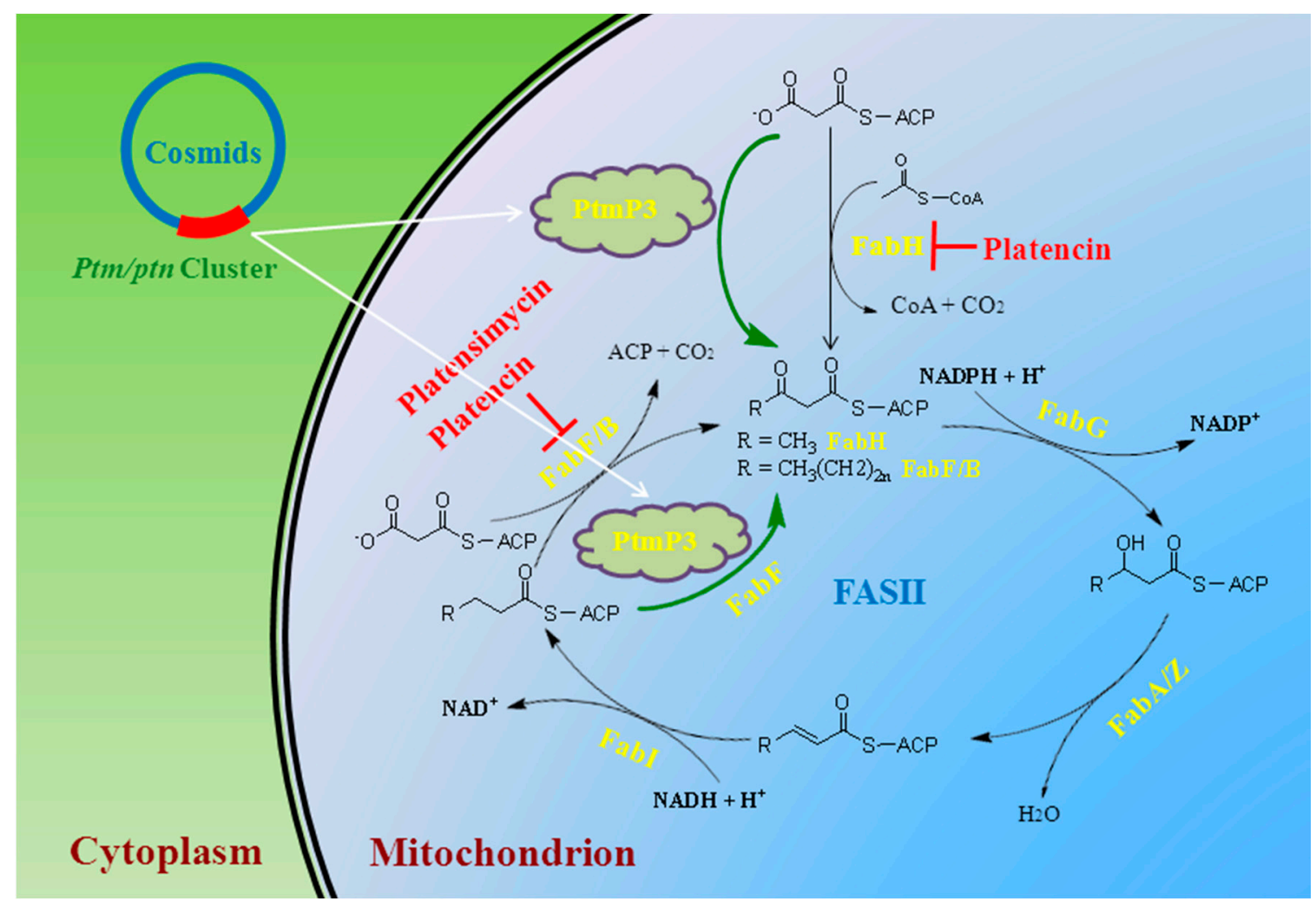

Figure 2. A diagram of the bacterial fatty acid synthesis cycle (FASII). Highlighted in red are (i) platensimycin inhibiting FabF/B and platencin dually inhibiting $\mathrm{FabF}$ and $\mathrm{FabH}$, and (ii) the two complementary mechanisms of platensimycin and platencin resistance in S. platensis by target replacement and target modification. Adapted from Peterson et al. [10].

\section{Recent Total Synthesis}

Structural studies revealed that platensimycin and platencin consist of a compact aliphatic tetracyclic ketolide core and polycyclic enone core, respectively, that were connected through a propionate tether to a highly oxygenated aromatic ring [9,22]. Therefore, the syntheses of platensimycin or platencin are generally divided into two sub-targets: the aromatic amine $\mathbf{3}$ and the carboxylic acid $\mathbf{4}$ or $\mathbf{6}$ constructed by tetracyclic cage $\mathbf{5}$ or Nicolaou's intermediate $\mathbf{7}$ are the key intermediates for synthesizing platensimycin or platencin, respectively (Scheme 1) [23]. Since the discovery of platensimycin and platencin, many concise synthetic routes targeting their complex molecular framework, especially the aliphatic tetracyclic ketolide core or polycyclic enone core, have been developed. 


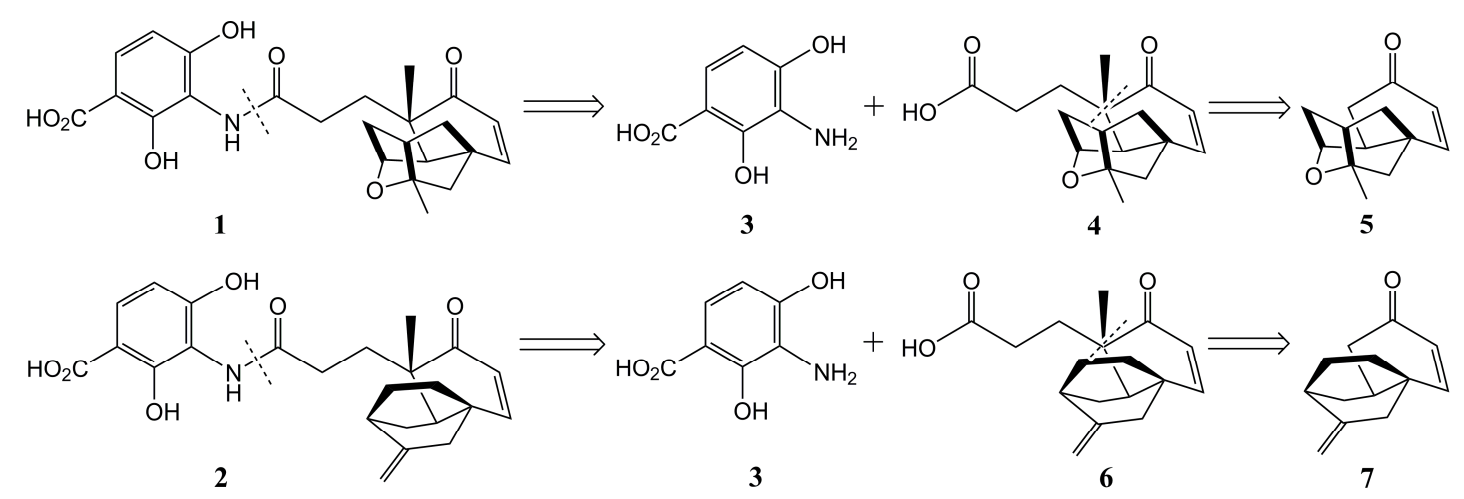

Scheme 1. The retrosynthetic analysis of platensimycin $\mathbf{1}$ and platencin $\mathbf{2}$.

\subsection{Zhu's Total Syntheses of (土)-Platensimycin and Platencinusing a Cascade Cyclization Approach}

Bifunctional Lewis acids are highly useful for developing cascade cyclization reactions because they can induce cyclization reactions via forming $\sigma$ - and/or $\pi$-complexes with the substrates, as well as the intermediate(s) generated in situ [24]. In 2012, a Chinese research group offered a new route for the synthesis of platensimycin (Scheme 2) using a mild and efficient bifunctional Lewis acid-induced cascade cyclization reaction for rapid construction of the tricyclic core of ent-kaurenoids [25]. With $\mathrm{ZnBr}_{2}$ as the bifunctional Lewis acid, enone $\mathbf{8}$ and diene $\mathbf{9}$ underwent cascade cyclization smoothly at room temperature and provided the tricyclic diketone $\mathbf{1 0}$ in one pot with good yields $(86 \%)$ and high diastereoselectivity. The resulting diketone $\mathbf{1 0}$ was converted to the di-tetramethylsilane (TMS) enol ether 11. Subsequent epoxidation using magnesium monoperoxyphthalate provided R-hydroxy ketone 12 selectively in $65 \%$ yield. The reduction of the ketone moiety of $\mathbf{1 2}$ followed by elimination of the resulting diol afforded $\mathbf{1 3}$ in good yields. Silyl enol formation followed by magnesium monoperoxyphthalate (MMPP) epoxidation of $\mathbf{1 3}$ provided R-hydroxyl ketone $\mathbf{1 4}$ as a single diastereomer, which can be equilibrated to the more stable R-hydroxyl ketone 15. Alcohol 15 was then acetylated and deacetoxylated using $\mathrm{SmI}_{2}$ to afford ketone 16. Finally, the reduction of ketone $\mathbf{1 6}$ with K-selectride followed by trifluoroacetic acid treatment afforded the Snider intermediate 17 (32\% overall yields in 11 steps), which could be converted to 1 according to the literature report [26,27].

Again using the Lewis acid induced cascade cyclization approach to platencin, with minor modifications (Scheme 3), Zhu et al. [28] synthesized the tricyclic diketone 10, which was further converted to enone 18 by oxidation. Then, enone 18 was reduced using Luche reduction conditions to diol 19 in a single diastereomer. The allylic alcohol of 19 was thus selectively oxidized and protected with $t$-butyldimethylsilyl (TBS) to afford enone 21, followed by stereoselective conversion to $\mathbf{2 3}$ via the Wharton transposition protocol. After protecting and functional group manipulations, compound $\mathbf{2 3}$ was converted to the bicycle octane 25 using Yoshimitsu's procedures. Finally, oxidative removal of the $p$-methoxybenzyl (PMB) ether followed by oxidation of the resulting alcohol completed the synthesis of Nicolaou's intermediate 7, which can also be converted to platencin according to the literature procedures [29].

The two synthesis routes employed the same cyclized product $\mathbf{1 0}$ as the precursor for the formal synthesis of Snider or Nicolaou's intermediates. However, the synthesis of Nicolaou's intermediate was 
a relatively short route than that for Snider intermediate, and finally gave a good overall yield for platencin ( $38 \%$ overall yields in 11 steps).

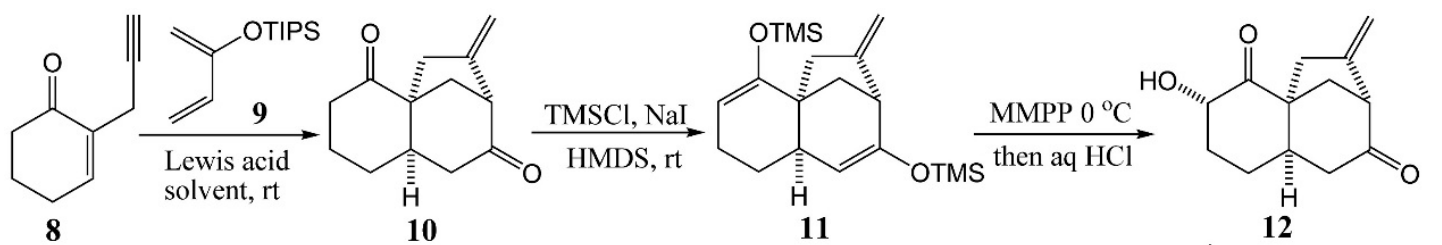

11

12

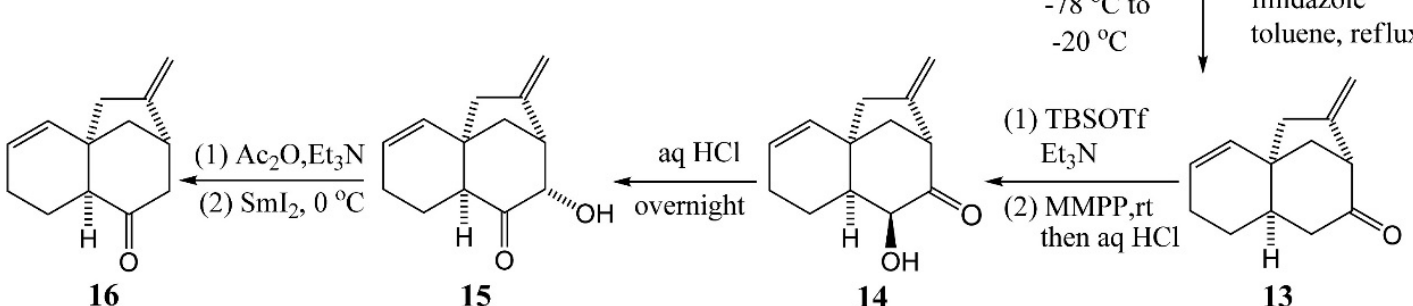

(1) $\mathrm{NaBH}_{4}, \mathrm{THF}(2) \mathrm{PPh}_{3}, \mathrm{I}_{2}$ $-78^{\circ} \mathrm{C}$ to $\quad$ Imidazole $-20^{\circ} \mathrm{C}$ toluene, reflux

(1) K-selectride $-78^{\circ} \mathrm{C}$ to rt (2) TFA, $0^{\circ} \mathrm{C}$

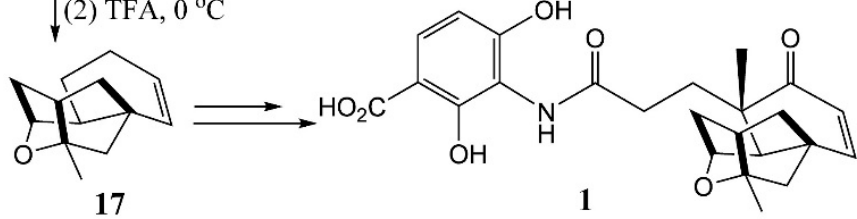

Scheme 2. A bifunctional Lewis acid induced cascade cyclization to the synthesis of platensimycin.
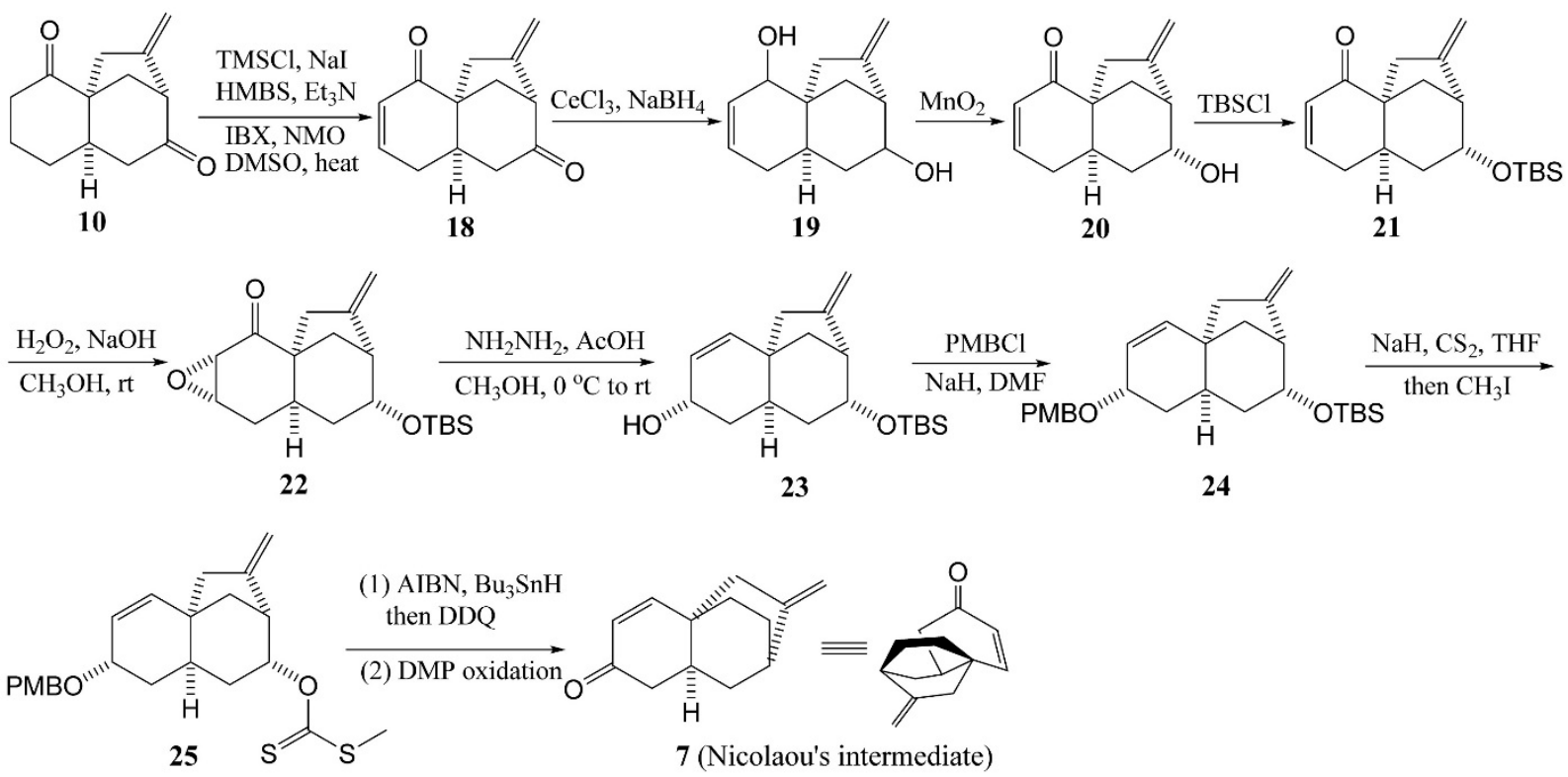

Scheme 3. Lewis acid induced cascade cyclization to the synthesis of platencin.

\subsection{Horii's Synthesis of Tetracyclic Cage 5}

To date, researchers have placed particular emphasis on the diverse approaches for chemical synthesis of the tetracyclic cage 5 because it is an important intermediate for approaching 1 [23]. As shown in Scheme 4 [30], epoxide 27 was readily obtained from 26 by a known three-step sequence, followed by regioselective trans-diaxial ring opening and dehydration of $\mathbf{2 7}$ to $\mathbf{2 8}$. The Diels-Alder reaction of $\mathbf{2 8}$ 
with Rawal's diene B gave $\mathbf{2 9}$ as a single isomer. The lactone 30, which was obtained by protection of the ketone functionality of $\mathbf{2 9}$, was treated with $\mathrm{MeMgCl}$ in tetrahydrofuran (THF)/hexamethylphosphoramide to give a diol intermediate, the secondary hydroxy group of which was then protected as its $t$-butyldimethylsilyl ether 31. The nitrile $\mathbf{3 1}$ was reduced with diisobutyl aluminum hydride to give aldehyde $\mathbf{3 2}$, and the subsequent addition of MeLi to $\mathbf{3 2}$ afforded $\mathbf{3 3}$ as an inconsequential 1:1 mixture of diastereomers. The ring closing metathesis (RCM) precursor 34 obtained by subjecting the diol 23 to an excess amount of Martin's sulfurane could be achieved efficiently by slowly adding the second-generation Grubbs catalyst in the presence of 1,4-benzoquinone at an elevated temperature to afford the tricyclic product 35. Finally, treatment of $\mathbf{3 5}$ with hydrochloric acid to remove the silyl and acetal protecting groups induced concurrent intramolecular etherification, providing tetracyclic cage $\mathbf{5}$. Although this was a new method for construction tricyclic diketone $\mathbf{2 9}$ as a single isomer, the preparation of the cyano lactone 28 inevitably prolonged the synthesis and gave a poor $(6.6 \%)$ overall yield for the tetracyclic cage 5.
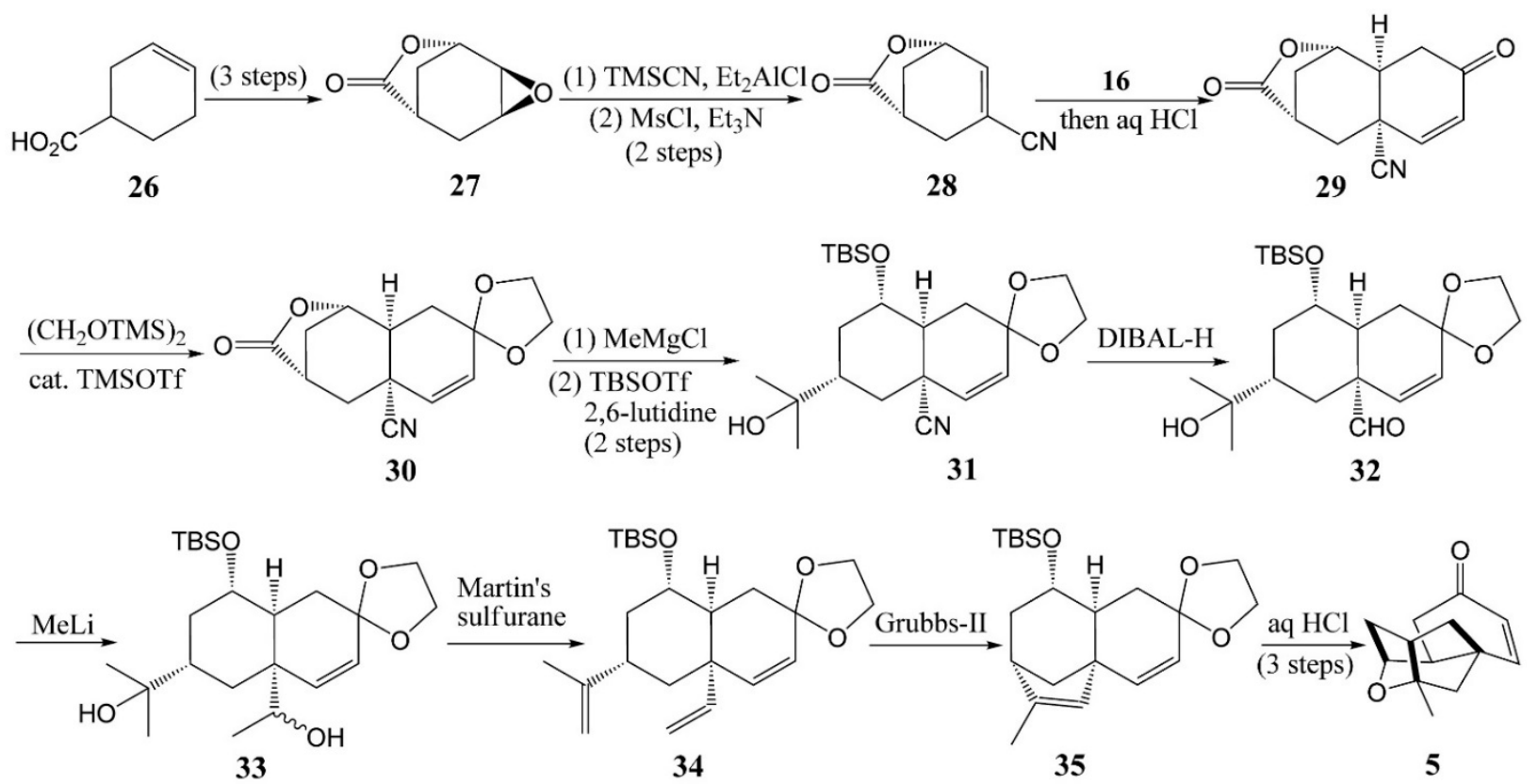

Scheme 4. Stereoselective synthesis of the tetracyclic cage 5.

\subsection{Gamal's Synthesis of Platencin}

Gamal et al. established a new route to platencin via decarboxylative radical cyclization, shown in Scheme 5 [31]. Enone 38 was prepared by adding Grignard reagent 37 to enone 36, followed by simple dehydration of the resultant tertiary alcohol. Then, enone $\mathbf{3 8}$ was converted to $\mathbf{3 9}$ under conventional sequential Michael reaction conditions. The introduction of oxygen functionality was conducted by $\mathrm{SeO}_{2}$-mediated propargylic oxidation leading to alcohol 40. Ketalization of the carbonyl group of $\mathbf{4 0}$ under azeotropic dehydration conditions furnished ketal 41, which was hydrolyzed with $\mathrm{LiOH}$ and further silylated with $t$-butyldi-methylsilyl chloride/imidazole to give the disilylated material $\mathbf{4 2}$. The decarboxylative radical cyclization of $\mathbf{4 2}$ then produced the desired compound $\mathbf{4 3}$. After hydrogenation with $\mathrm{PtO}_{2}$ in the presence of triethylamine, compound $\mathbf{4 3}$ was converted to a saturated product, and its ketal and the silyl ether were sequentially cleaved using $1 \mathrm{~N} \mathrm{HCl}$ followed by tetrabutylammonium 
fluoride to provide hydroxyketone 44. Hydroxyketone 44 was then subjected to Tebbe olefination followed by Parikh-Doering oxidation to produce ketone 45. The conventional phenylselenylation of ketone 45 with lithium diisopropylamide/phenylselenyl chloride, followed by oxidative elimination with $\mathrm{H}_{2} \mathrm{O}_{2}$, successfully gave enone $\mathbf{4 6}$ which could be converted to platencin [29].

The decarboxylative radical cyclization of alkynyl silyl ester $\mathbf{4 2}$ was the key step for constructing the tricyclic core of platencin. With $\mathrm{Pb}(\mathrm{OAc})_{4}$ in the presence of pyridine in refluxing 1,4-dioxane, the key decarboxylation allowed the rapid construction of the twisted polycyclic compound $\mathbf{4 3}$, but with a poor $(30 \%)$ yeild.

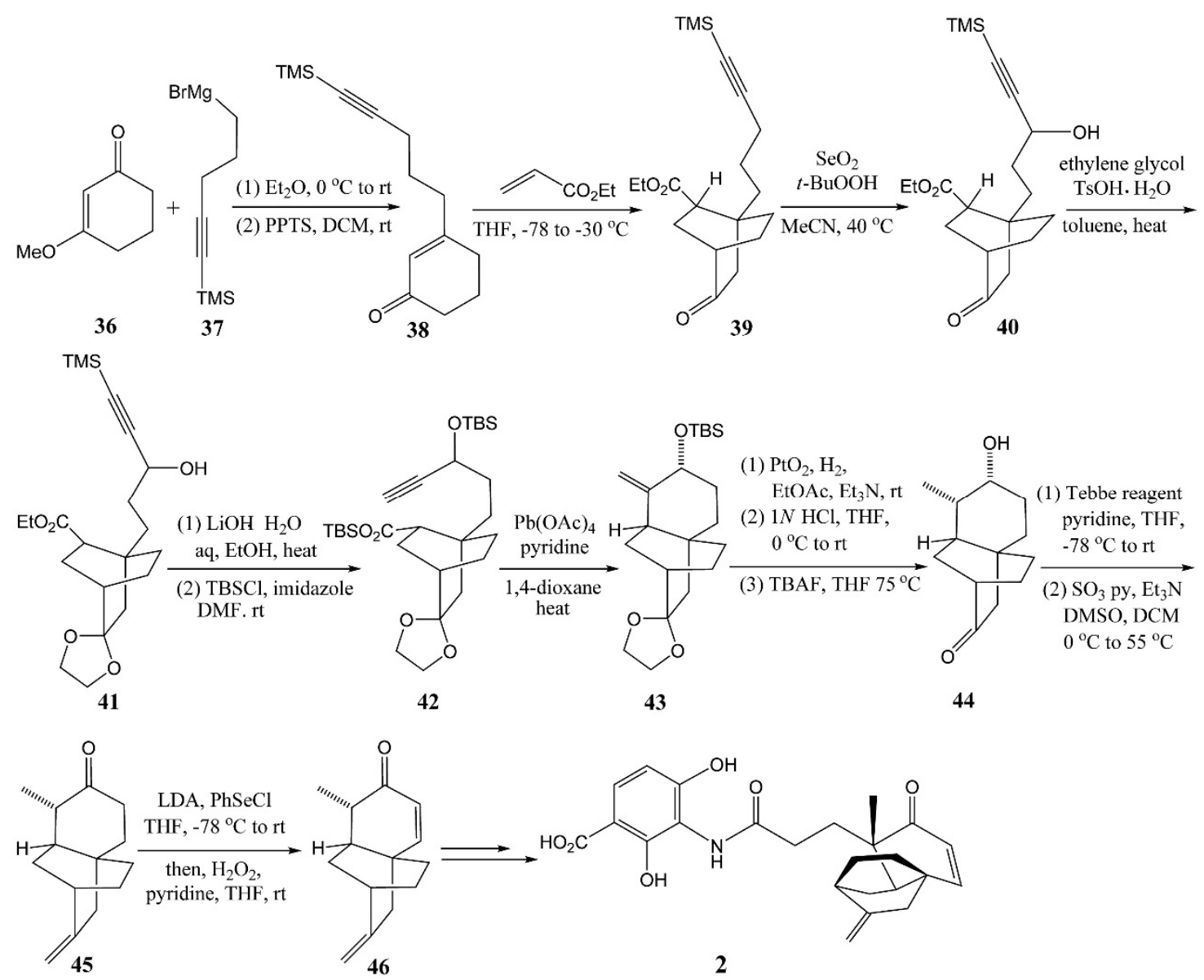

Scheme 5. Synthesis of platencin via decarboxylative radical cyclization.

\subsection{Chang's Synthesis of Platencin}

Chang et al. used an intramolecular Diels-Alder (IMDA) approach for the direct assembly of the tricarboxylic framework associated with platencin, outlined in Scheme 6 [32].

The commercially available amino-diol 47 was converted into 1,3-dioxane 48 under the conditions defined by Nordin and Thomas [33]. The condensation of 48 with aldehyde 49 in the presence of trifluoroacetic anhydride gave the imine 50, which was deprotonated using lithium diisopropylamide. The resulting anion was alkylated with the benzyl ether $\mathbf{5 1}$, followed by an acidic workup that gave the desired aldehyde 52. Treating compound 52 with ethynylmagnesium bromide in THF gave the anticipated propargyl alcohol 53. The sequential treatment of compound $\mathbf{5 3}$ with the readily prepared Lewis acidic hydrostannane $\mathrm{Bu}_{2} \mathrm{Sn}(\mathrm{OTf}) \mathrm{H}$ followed by $n \mathrm{BuLi}$ gave the required Z-configured alkene 54 . 
The tetraene 56 was obtained as an approximately 1:1 mixture of diastereoisomers by the treatment of an approximately 1:1 mixture of $\mathbf{5 4}$ and acetonide 55 with $\left[\mathrm{Pd}\left(\mathrm{PPh}_{3}\right)_{4}\right]$ in the presence of $\mathrm{CuI}$ and $\mathrm{CsF}$. Compound 56 was oxidized to the corresponding ketone 57 using the Dess-Martin periodinane in the presence of pyridine. Then, the anticipated intramolecular Diels-Alder adduct $\mathbf{5 8}$ was obtained from $\mathbf{5 7}$ under-reflux in toluene. Compound $\mathbf{5 8}$ was exposed to dihydrogen in the presence of 5\% $\mathrm{Pd}$ to give the crystalline ketal 59, which was oxidized with pyridinium chlorochromate to give carboxylic acid 60. Cleavage of 60 gave the diol 61, which could be regioselectively monooxidized using the sterically demanding oxammonium salt to afford acyloin 62. After esterification, the acyloin $\mathbf{6 2}$ was converted to benzoate 63 and subsequently generated the diketo ester 64 through a samarium iodide-promoted reduction reaction. Subjecting compound 64 to a Wittig olefination reaction using $\mathrm{Ph}_{3} \mathrm{P}=\mathrm{CH}_{2}$ gave the desired alkene $\mathbf{6 5}$, which could finally be converted to $\mathbf{6}$ by the saponification of ester $\mathbf{6 5}$ using aqueous sodium hydroxide in THF.
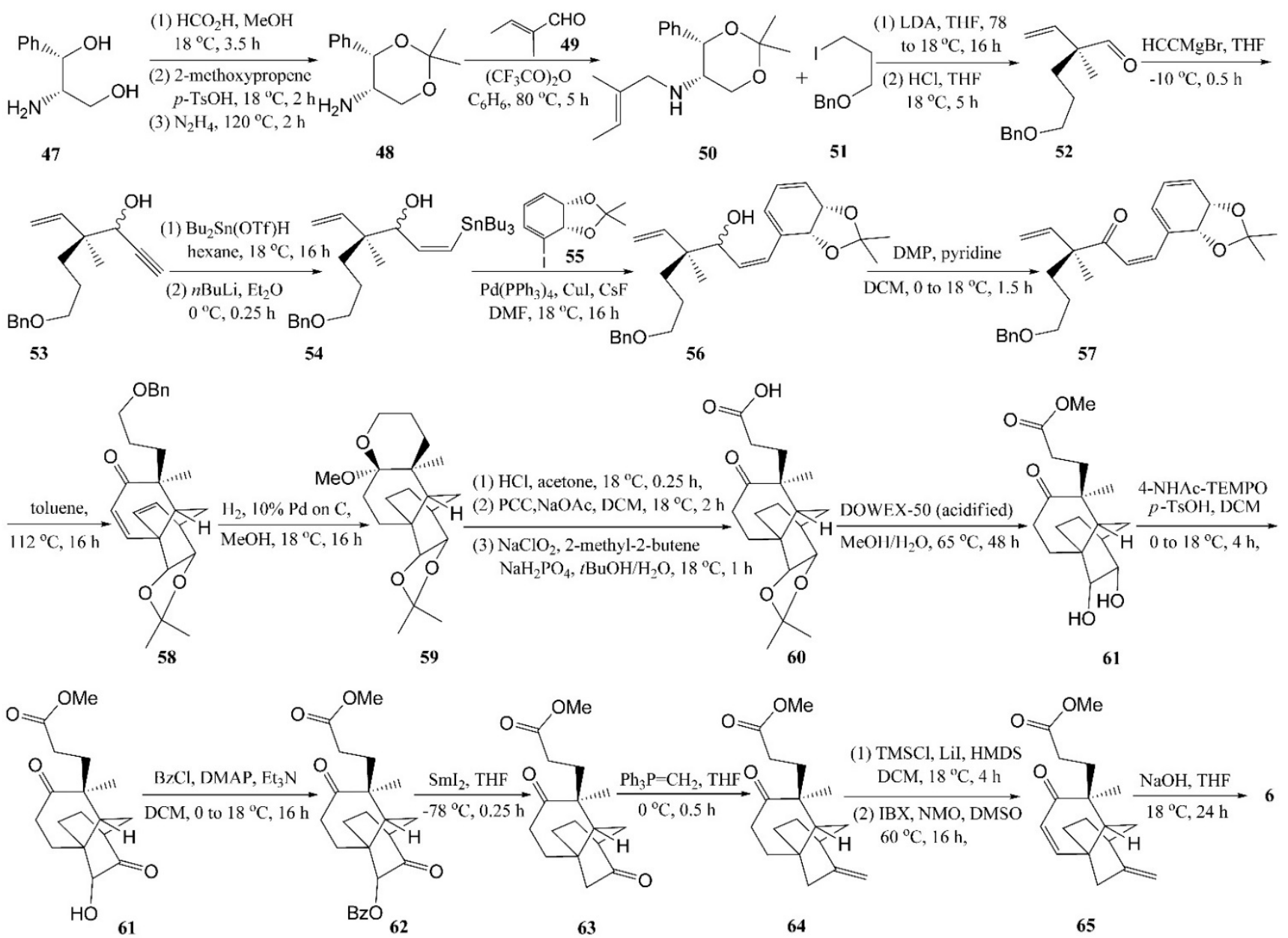

Scheme 6. Synthesis the tricarbocyclic framework of platencin using an intramolecular Diels-Alder approach.

Although the original total synthesis of platencin reported by Nicolaou and co-workers in 2008 [29] used the same IMDA approach to establish the framework 7 in an enantioselective manner, the pivotal aspect of this work was the use of the enantiomerically pure cis-1,2-dihydrocatechol (which is obtained by the whole-cell biotransformation of iodobenzene using a genetically engineered form of $E$. coli) as 
the starting material [32]. Furthermore, derivatives of enone 7 that already incorporated a suitably constituted quaternary carbon center adjacent to the carbonyl residue and thus were more readily elaborated to (-)-platencin, were generated by this modified route in as highly controlled a manner as possible.

\section{Recent Analogues and Their Antibacterial Activities}

Since the discovery of platensimycin and platencin, significant effort has been undertaken to prepare platensimycin and platencin analogues with high antibacterial activities by organic synthesis. Meanwhile, structure activity relationship (SAR) studies were performed to find more effective analogues with high efficiency. The SAR studies have revealed that modifications on the 2,4-dihydroxybenzoic acid moiety of platensimycin or platencin usually diminish most of the biological activity of this antibiotic [34-36]. In sharp contrast, decorations or modifications on the caged ring often retain good biological activity [34,37].

Because the tetracyclic/tricyclic core of platensimycin or platencin is relatively difficult to prepare chemically, several groups have focused their attention on the replacement of the "difficult-to-synthesize" moiety [38].

A combinatorial strategy in which dihydroxybenzoic acid was coupled to a variety of alkyl chains was developed by Wang et al. [38] based on the finding that FAS enzymes utilize substrates with long alkyl chains to make fatty acids and the idea that there ought to be a hydrophobic pocket at or near the active site of these enzymes. They designed and synthesized a series of 2,4-dihydroxybenzoic acid esters with a terminal amino group. From bioactivity studies, only compound $\mathbf{6 6}$ showed excellent in vitro antibacterial activity against $B$. subtilis (3160), MRSA and VRE, with MIC values of 4, 16 and $8 \mu \mathrm{g} / \mathrm{mL}$, respectively (Figure 3).

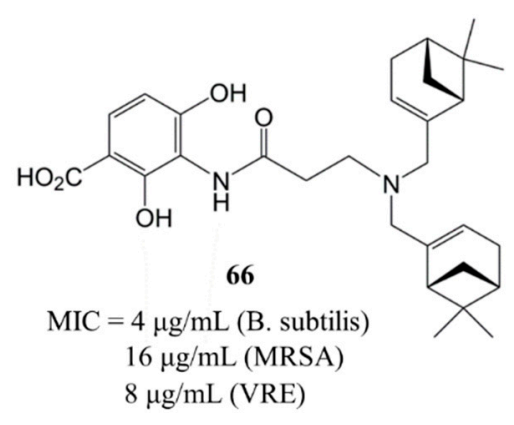

Figure 3. Structure and antibacterial activity (MIC, $\mu \mathrm{g} / \mathrm{mL}$ ) of compound 66.

Plesch's group also focused on the development of novel simplified platensimycin analogues and thereby synthesized a series of compounds containing the anilide group and the ketone, both identified as essential for binding of the active components to the Fab F complex [39]. After a standardized agar diffusion assay, ketones $\mathbf{6 7}$ and $\mathbf{6 8}$ showed a broad spectrum of antibacterial activity, comparable to the antibiotics tetracycline and clotrimazol (Table 1). 
Table 1. Agar diffusion assay for compounds 67, 68, tetracycline and clotrimazol (50 mg/disc).

\begin{tabular}{ccccc}
\multicolumn{1}{c}{} & & & \\
\hline Strains & $\mathbf{6 7}: \mathbf{R}=\mathbf{P h}$ & $\mathbf{6 8 :} \mathbf{R}=$ Trans $\mathbf{H}_{3} \mathbf{C}-\mathbf{C H}=\mathbf{C H}-$ & Tetracycline & Clotrimazol \\
\hline Escherichia coli & 11 & 10 & 25 & 0 \\
Pseudomonas antimicrobia & 14 & 10 & 23 & 0 \\
Staphylococcus equorum & 0 & 11 & 23 & 9 \\
Streptococcus entericus & 16 & 14 & 12 & 11 \\
Candida glabrata & 20 & 17 & $\mathrm{nt}$ & 15 \\
Aspergillus niger & 0 & 6 & $\mathrm{nt}$ & 15 \\
Yarrowia lipolytica & 0 & 7 & $\mathrm{nt}$ & 20 \\
Hyphopichia burtonii & 10 & 15 & $\mathrm{nt}$ & 17 \\
\hline
\end{tabular}

Zones of inhibition (in $\mathrm{mm}$ ); nt, not tested; 0 , no measurable zone of inhibition.

Fisher et al. described an approach to platensimycin analogue design using structure-based ligand design (SBLD), a powerful approach that can facilitate the discovery of bioactive small molecules when high quality structural information is available [40]. They designed and synthesized a series of platensimycin analogous and determined the affinities of these compounds for the C163Q mutant of FabF using a WaterLOGSY [41] competition binding assay. The resulting designed compounds were shown to bind in the platensimycin binding site of the C163Q mutant of FabF, and, in several cases, compounds 69, 70, 71 and 72 had higher affinity (lower dissociation constants) than the reference compound $\mathbf{7 3}$ (Table 2).

Table 2. Dissociation constants of compounds 69, 70, 71, 72 and reference compound 73 for the C163Q mutant of FabF.

\begin{tabular}{|c|c|c|c|}
\hline Structure & $\mathbf{R}$ & Compounds & $\mathrm{Kd} / \mu \mathrm{M}$ \\
\hline \multirow{4}{*}{$\mathrm{OH}$} & $-\mathrm{SO}_{2} \mathrm{NHBOC}$ & 69 & $50 \pm 10$ \\
\hline & $-\mathrm{SO}_{2} \mathrm{NH}_{2}$ & 70 & $100 \pm 20$ \\
\hline & $-\mathrm{CONHiPr}$ & 71 & $110 \pm 30$ \\
\hline & 3-pyridyl & 72 & $120 \pm 20$ \\
\hline & & 73 & $650 \pm 90$ \\
\hline
\end{tabular}

\section{Conclusions}

Multidrug resistance has become a serious global concern. Research on new antibacterial drugs that are effective against resistant pathogenic bacteria is highly crucial. This article describes the efforts of chemists to overcome multidrug resistance in the case of the new antibiotic platensimycin isolated from extracts of Streptomyces platensis, and its analogue platencin. These natural products interfere with the synthesis of fatty acids by selectively inhibiting -FabF, and are of particular interest because they exhibit a new mode of action that overcomes existing drug resistance. Antibacterial studies have shown that 
these new antimicrobials have great potential in inhibiting MRSA, VRE, VISA and penicillin-resistant Streptococcus pneumoniae. The fascinating molecular architecture, novel mode of action and high antibacterial activities have inspired many different approaches to the synthesis and chemical modifications of platensimycin and platencin in a relatively short time.

\title{
Acknowledgments
}

This work was supported by grants from Basic Scientific Research Funds in Central Agricultural Scientific Research Institutions (No. 1610322014003) and The Agricultural Science and Technology Innovation Program (CAAS-ASTIP-2014-LIHPS-04).

\section{Author Contributions}

The manuscript was conceived by all authors. Jianping Liang, Yunpeng Yi, Yu Liu collected the documents and wrote the draft manuscript which was subsequently edited by Ruofeng Shang and Jiatu Wang.

\section{Conflicts of Interest}

The authors declare no conflict of interest.

\begin{abstract}
Abbreviation
Fatty acid acyl carrier protein synthases II: FabF; Fatty acid acyl carrier protein synthases III: FabH; Methicillin-resistant Staphylococcus aureus: MRSA; Vancomycin-intermediate S. aureus: VISA; Vancomycin-resistant Enterococci: VRE; High performance liquid chromatography: HPLC; Nuclear magnetic resonance: NMR; Escherichia coli: E. coli; Minimal inhibitory concentration: MIC; Type II bacterial fatty acid synthesis: FASII; Tetramethylsilane: TMS; Magnesium monoperoxyphthalate: MMPP; $t$-Butyldimethylsilyl: TBS; $p$-Methoxybenzyl: PMB; Tetrahydrofuran: THF; Ring closing metathesis: RCM; Intramolecular Diels-Alder: IMDA; Structure activity relationship: SAR.
\end{abstract}

\section{References}

1. Spicknall, I.H.; Foxman, B.; Marrs, C.F.; Eisenberg, J.N. A Modeling Framework for the Evolution and Spread of Antibiotic Resistance: Literature Review and Model Categorization. Am. J. Epidemiol. 2013, 178, 508-520.

2. Brown, D.G.; May-Dracka, T.L.; Gagnon, M.M.; Tommasi, R. Trends and exceptions of physical properties on antibacterial activity for gram-positive and gram-negative pathogens. J. Med. Chem. 2014, 57, 10144-10161.

3. Shang, R.F.; Wang, J.T.; Guo, W.Z.; Liang, J.P. Efficient antibacterial agents: A review of the synthesis, biological evaluation and mechanism of pleuromutilin derivatives. Curr. Top. Med. Chem. 2013, 13, 3013-3025.

4. Wang, J.; Kodali, S.; Lee, S.H.; Galgoci, A.; Painter, R.; Dorso, K.; Racine, F.; Motyl, M.; Hernandez, L.; Tinney, E.; et al. Discovery of platencin, a dual FabF and FabH inhibitor with in vivo antibiotic properties. Proc. Natl. Acad. Sci. USA. 2007, 104, 7612-7616. 
5. Kirst, H.A. Developing new antibacterials through natural product research. Expert Opin. Drug Discv. 2013, 8, 479-493.

6. Wang, J.; Soisson, S.M.; Young, K.; Shoop, W.; Kodali, S.; Galgoci, A.; Painter, R.; Parthasarathy, G.; Tang, Y.; Cummings, R.; et al. Platensimycin is a selective FabF inhibitor with potent antibiotic properties. Nature 2006, 441, 358-361.

7. Young, K.; Jayasuriya, H.; Ondeyka, J.G.; Herath, K.; Zhang, C.; Kodali, S.; Galgoci, A.; Painter, R.; Brown-Driver, V.; Yamamoto, R.; et al. Discovery of FabH/FabF Inhibitors from Natural Products. Antimicrob. Agents Chemother. 2006, 50, 519-526.

8. Zhang, C,W.; Ondeyka, J.; Herath, K.; Jayasuriya, H.; Guan, Z.Q.; Zink, D.L.; Dietrich, L.; Burgess, B.; Ha, S.N.; Wang, J.; et al. Platensimycin and platencin congeners from Streptomyces platensis. J. Nat. Prod. 2011, 74, 329-340.

9. Martens, E.; Demain, A.L. Platensimycin and platencin: Promising antibiotics for future application in human medicine. J. Antibiot. 2011, 64, 705-710.

10. Peterson, R.M.; Huang, T.; Rudolf, J.D.; Smanski, M.J.; Shen, B. Mechanisms of self-resistance in the platensimycin- and platencin- producing Streptomyces platensis MA7327 and MA7339 strains. Chem. Bio. 2014, 21, 389-397.

11. Singh, S.B.; Jayasuriya, H.; Ondeyka, J.G.; Herath, K.B.; Zhang, C.; Zink, D.L.; Tsou, N.N.; Ball, R.G.; Basilio, A.; Genilloud, O.; et al. Isolation, Structure, and Absolute Stereochemistry of Platensimycin, A Broad Spectrum Antibiotic Discovered Using an Antisense Differential Sensitivity Strategy. J. Am. Chem. Soc. 2006, 128, 11916-11920.

12. Habich, D.; von Nussbaum, F. Platensimycin, a new antibiotic and "superbug challenger" from nature. Chem. Med. Chem. 2006, 1, 951-954.

13. Barton, S. New antibiotic on the horizon? Nat. Rev. Microbiol. 2006, 4, doi:10.1038/nrd2098.

14. Palanichamy, K.; Kaliappan, K.P. Discovery and syntheses of "superbug challengers"platensimycin and platencin. Chem. Asian J. 2010, 5, 668-703.

15. Singh, S.B.; Young, K. New antibiotic structures from fermentations. Expert Opin.Ther. Pat. 2010, $20,1359-1371$.

16. Jayasuriya, H.; Herath, K.B.; Zhang, C.; Zink, D.L.; Basilio, A.; Genilloud, O.; Diez, M.T.; Vicente, F.; Gonzalez, I.; Salazar, O.; et al. Isolation and Structure of Platencin: A FabH and FabF Dual Inhibitor with Potent Broad-Spectrum Antibiotic Activity. Angew. Chem. 2007, 46, 4768-4772.

17. Herath, K.B.; Attygalle, A.B.; Singh, S.B. Biosynthetic studies of platensimycin. J. Am. Chem. Soc. 2007, 129, 15422-15423.

18. Jayasuriya, H.; Herath, K.B.; Ondeyka, J.G.; Zink, D.L.; Burgess, B.; Wang, J.; Singh, S.B. Structure of homoplatensimide A: A potential key biosynthetic intermediate of platensimycin isolated from Streptomyces platensis. Tetrahedron Lett. 2008, 49, 3648-3651.

19. Smanski, M.J.; Yu, Z.; Casper, J.; Lin, S.; Peterson, R.M.; Chen, Y.; Wendt-Pienkowski, E.; Rajski, S.R.; Shen, B. Dedicated ent-kaurene and ent-atiserene synthases for platensimycin and platencin biosynthesis. Proc. Natl. Acad. Sci. USA. 2011, 108, 13498-13503.

20. Payne, D.J.; Warren, P.V.; Holmes, D.J.; Ji, Y.; Lonsdale, J.T. Bacterial fatty-acid biosynthesis: A genomics-driven target for antibacterial drug discovery. Drug Disc. Today 2001, 6, 537-544.

21. Hopwood, D.A. How do antibiotic-producing bacteria ensure their self-resistance before antibiotic biosynthesis incapacitates them? Mol. Microbiol. 2007, 63, 937-940. 
22. McGrath, N.A.; Bartlett, E.S.; Sittihan, S.; Njardarson, J.T. A concise ring-expansion route to the compact core of platensimycin. Angew. Chem. Int. Ed. Engl. 2009, 48, 8543-8546.

23. Saleem, M.; Hussain, H.; Ahmed, I.; van Ree, T.; Krohn, K. Platensimycin and its relatives: A recent story in the struggle to develop new naturally derived antibiotics. Nat. Prod. Rep. 2011, 28, 1534-1579.

24. Yamamoto, Y. From sigma- to pi- electrophilic Lewis acids. Application to selective organic transformations. J. Org. Chem. 2007, 72, 7817-7831.

25. Zhu, L.Z.; Han, Y.J.; Du, G.Y.; Lee, C.S. A bifunctional Lewis Acid induced cascade cyclization to the tricyclic core of ent-kaurenoids and its application to the formal synthesis of ( \pm )-platensimycin. Org. Lett. 2013, 15, 524-527.

26. Nicolaou, K.C.; Li, A.; Edmonds, D.J. Total synthesis of platensimycin. Angew. Chem. Int. Ed. Engl. 2006, 45, 7086-7090.

27. Zou, Y.; Chen, C.H.; Taylor, C.D.; Foxman, B.M.; Snider, B.B. Formal synthesis of (+/-)-platensimycin. Org. Lett. 2007, 9, 1825-1828.

28. Zhu, L.Z.; Zhou, C.S.; Yang, W.; He, S.Z.; Cheng, G.J.; Zhang, X.H.; Lee, C.S. Formal syntheses of $( \pm)$-platensimycin and $( \pm)$-platencin via a dual-mode Lewis acid induced cascade cyclization approach. J. Org. Chem. 2013, 78, 7912-7929.

29. Nicolaou, K.C.; Tria, G.S.; Edmonds, D.J. Total synthesis of platencin. Angew. Chem. Int. Ed. Engl. 2008, 47, 1780-1783.

30. Horii, S.; Torihata, M.; Nagasawa, T.; Kuwahara, S. Stereoselective approach to the racemic oxatetracyclic core of platensimycin. J. Org. Chem. 2013, 78, 2798-2801.

31. Moustafa, G.A.; Saku, Y.; Aoyama, H.; Yoshimitsu, T. A new route to platencin via decarboxylative radical cyclization. Chem. Commun. 2014, 50, 15706-15709.

32. Chang, E.L.; Schwartz, B.D.; Draffan, A.G.; Banwell, M.G.; Willis, A.C. A chemoenzymatic and fully stereo-controlled total synthesis of the antibacterial natural product ( \pm )-platencin. Chem. Asian. J. 2015, 10, 427-439.

33. Nordin, I.C.; Thomas, J.A. An improved synthesis of (4S,5S)-2,2-dimethyl-4-phenyl-1,3-dioxan-5amine Tetrahedron Lett. 1984, 25, 5723-5724.

34. Nicolaou, K.C.; Stepan, A.F.; Lister, T.; Li, A.; Montero, A.; Tria, G.S.; Turner, C.I.; Tang, Y.; Wang, J.; Denton, R.M.; et al. Design, synthesis, and biological evaluation of platensimycin analogues with varying degrees of molecular complexity. J. Am. Chem. Soc. 2008, 130, 13110-13119.

35. Wang, J.; Lee, V.; Sintim, H.O. Efforts towards the Identification of Simpler Platensimycin Analogues-The Total Synthesis of Oxazinidinyl Platensimycin. Chem. Eur. J. 2009, 15, 2747-2750.

36. Tiefenbacher, K.; Gollner, A.; Mulzer, J. Syntheses and antibacterial properties of iso-platencin, Cl-iso-platencin and Cl-platencin: Identification of a new lead structure. Chem. Eur. J. 2010, 16, 9616-9622.

37. Shen, H.C.; Ding, F.X.; Singh, S.B.; Parthasarathy, G.; Soisson, S.M.; Ha, S.N.; Chen, X.; Kodali, S.; Wang, J.; Dorso, K.; et al. Synthesis and biological evaluation of platensimycin analogs. Bioorg. Med. Chem. Lett. 2009, 19, 1623-1627. 
38. Wang, J.X.; Sintim, H.O. Dialkylamino-2,4-dihydroxybenzoic acids as easily synthesized analogues of platensimycin and platencin with comparable antibacterial properties. Chem. Eur. J. 2011, 17, 3352-3357.

39. Plesch, E.; Bracher, F.; Krauss, J. Synthesis and Antimicrobial Evaluation of Novel Platensimycin Analogues. Arch. Pharm. 2012, 345, 657-662.

40. Fisher, M.; Basak, R.; Kalverda, A.P.; Fishwick, C.W.; Turnbull, W.B.; Nelson, A. Discovery of novel FabF ligands inspired by platensimycin by integrating structure-based design with diversity-oriented synthetic accessibility. Org. Biomol. Chem. 2014, 12, 486-494.

41. Dalvit, C.; Fasolini, M.; Flocco, M.; Knapp, S.; Pevarello, P.; Veronesi, M. NMR-Based screening with competition water-ligand observed via gradient spectroscopy experiments: Detection of high-affinity ligands. J. Med. Chem. 2002, 45, 2610-2614.

(C) 2015 by the authors; licensee MDPI, Basel, Switzerland. This article is an open access article distributed under the terms and conditions of the Creative Commons Attribution license (http://creativecommons.org/licenses/by/4.0/). 